\title{
The Source of Semiprimeness of Semigroups
}

\author{
Barış Albayrak, ${ }^{1}$ Didem Yeşil $\mathbb{D}^{2}{ }^{2}$ and Didem Karalarlioğlu Camci ${ }^{2}$ \\ ${ }^{1}$ Çanakkale Onsekiz Mart University, Biga Faculty of Applied Sciences, Department of Banking and Finance, Çanakkale, Turkey \\ ${ }^{2}$ Çanakkale Onsekiz Mart University, Faculty of Arts and Sciences, Department of Mathematics, Çanakkale, Turkey \\ Correspondence should be addressed to Didem Yeşil; dyesil@comu.edu.tr
}

Received 23 August 2020; Revised 3 February 2021; Accepted 11 May 2021; Published 2 June 2021

Academic Editor: Feng Feng

Copyright ( 2021 Barış Albayrak et al. This is an open access article distributed under the Creative Commons Attribution License, which permits unrestricted use, distribution, and reproduction in any medium, provided the original work is properly cited.

In this study, we define new semigroup structures using the set $S_{S}=\{a \in S \mid a S a=0\}$ which is called the source of semiprimeness for a semigroup $S$ with zero element. $\left|S_{S}\right|$-idempotent semigroup, $\left|S_{S}\right|$-regular semigroup, $\left|S_{S}\right|$-reduced semigroup, and $\left|S_{S}\right|$-nonzero divisor semigroup which are generalizations of idempotent, regular, reduced, and nonzero divisor semigroups in semigroup theory are investigated, and their basic properties are determined. In addition, we adapt some well-known results in semigroup theory to these new semigroups.

\section{Introduction}

Semiprime ideals play a very important role in semigroups. Since every ring can be considered as a semigroup under multiplication, we have more generalized theorems of [1]. The aim of this study is to obtain new semigroup structures by using the definition of semiprimeness in semigroups in the sense of the study by Aydın et al. [1]. There are different equivalent definitions of semiprimeness. One of these is, if $a S a=0$ with $a \in S$ implies $a=0$, then $S$ is called a semiprime semigroup. In [1], the authors have defined the set $S_{R}=$ $\{a \in R \mid a R a=0\}$ called source of semiprimeness of ring $R$ by using the ring version of semiprimeness definition. In addition, they have obtained new ring and field structures. In this study, new semigroup structures have been obtained by using the source set of semiprimeness of semigroups. These structures are investigated, and some results of the semigroup theory are adapted to the new semigroups.

\section{Preliminaries}

In semigroup theory, there are many studies on different semigroup types. Adams examined the properties of semiprime semigroups in [2]. In [3], Van Rooyen worked on the ideals of semigroups. In [4-6], the authors have worked on reduced, regular, and zero-divisor semigroups. This article will bring a different perspective to these various semigroups.

First, let us give the definitions mentioned in this article which are frequently used in semigroup theory. The studies by Clifford and Preston and Grillet [7, 8] are used for definitions.

Let $(S, \cdot)$ be a semigroup. A zero element of a semigroup $S$ is an element $0 \in S$, such that $0 \cdot a=a \cdot 0=0$ for all $a \in S$. Throughout this study, $S$ will be taken as a semigroup with zero element. The $e \in S$ element, such that $e^{2}=e$, is called an idempotent element. The semigroup whose all elements are idempotent is called an idempotent semigroup. An element $x$ of a semigroup $S$ is called regular element if there exist at least one $y \in S$, such that $x y x=x$. The semigroup whose all elements are regular is called a regular semigroup. For $a \in S$, if there exist $n \in \mathbb{Z}^{+}$, such that $a^{n}=0$ and $a^{n-1} \neq 0$, then $a$ is called nilpotent. A semigroup without nilpotent elements other than zero is called a reduced semigroup. An element $x$ of a semigroup $S$ is called zero divisor element if there exist $0 \neq y, 0 \neq z \in S$, such that $x y=0$ (left zero divisor) and $z x=$ 0 (right zero divisor). $S$ is called the nonzero divisor semigroup, if there is no nonzero zero divisor element in $S$.

Now, let us give the definitions of ideal and semiprime semigroup which are the basis of our article. From [2, 9], the subset $I$ of semigroup $S$ is called an ideal if IS $\subseteq I$ (right ideal) and SI $\subseteq I$ (left ideal). One of the equivalent definition 
of the semiprime semigroup, which using this study, is given as follows: if $a S a=0$ with $a \in S$ implies $a=0$, then $S$ is called the semiprime semigroup.

\section{Results of Different Types of $\left|S_{S}\right|$ - Semigroups}

Definition 1. Let $S$ be a semigroup with zero and $A$ be a nonempty subset of $S$. The subset of $S$,

$$
S_{S}(A)=\{a \in S \mid a A a=0\},
$$

is called the source of semiprimeness of $A$ in $S$. For semigroup $S$, the notation $S_{S}$ is used instead of $S_{S}(S)$. Then, the source of semiprimeness of the $S$ semigroup is defined as follows:

$$
S_{S}=\{a \in S \mid a S a=0\} .
$$

Now, let us examine the basic properties of the source of semiprimeness of semigroup $S$. In [1], the set of source of semiprimeness was investigated for the rings. The properties that can be exactly provided for semigroups are given with reference without proof in this study. First of all, let us mention some facts that can be easily seen but useful for understanding the set.

(1) For every semigroup with zero, since $0 \in S_{S}(A)$, $S_{S}(A) \neq \varnothing$ is provided for every subset $A$ of $S$

(2) It is easy to see that $S_{A} \subseteq S_{S}(A)$ for the subsemigroup $A$ of $S$

(3) For subsets $A$ and $B, A \subseteq B$ implies $S_{S}(B) \subseteq S_{S}(A)$ ([1], Proposition 2.2)

Now, let us investigate the properties of idempotent, regular, nilpotent, and zero divisor elements. These properties form the basis for defining the new semigroup structures that we will define in the next section. By using the results obtained, we will obtain the definitions of the $\left|S_{S}\right|$-idempotent semigroup, $\left|S_{S}\right|$-regular semigroup, $\left|S_{S}\right|$-reduced semigroup, and $\left|S_{S}\right|$-nonzero divisor semigroup.

Lemma 1. Let $S$ be a semigroup, e be an idempotent element, and $x$ be a regular element, such that $x y x=x$ for $y \in S$. Then, the following properties are provided.

(1) $e S_{S} \subseteq S_{e S}$

(2) $x y S_{S} \subseteq S_{x y S}$

(3) If $S$ is an idempotent semigroup, then $S_{S}=\{0\}$

(4) If $S$ is a regular semigroup, then $S_{S}=\{0\}$

(5) If $a \in S_{S}$, then $a$ is a nilpotent element

(6) If $a \in S_{S}$, then $a$ is a zero divisor element

Proof

(1) Let $e a \in e S_{S}$. Then, since $a \in S_{S}$, we write $a S a=0$. From this equation, we get

$$
e a(e S) e a=e a(e S e) a \subseteq e(a S a)=\{0\} .
$$

This gives us $e a \in S_{e S}$. So, $e S_{S} \subseteq S_{e S}$ is provided.

(2) For regular element $x$, such that $x y x=x$ for $y \in S$, this implies that $x y x y=x y$, and $x y$ is an idempotent element. From (1), it is clear that $x y S_{S} \subseteq S_{x y S}$.

(3) Let $S$ be an idempotent semigroup and $a \in S_{S}$. Since $a S a=0$, the equation $a a a=0$ is satisfied for $a \in S$. Hence, using $a$ as an idempotent element, we get $a=0$. Then, $S_{S}=\{0\}$.

(4) Let $S$ be a regular semigroup and $x \in S_{S}$. In this case, $x S x=0$, and there exist $y \in S$, such that $x y x=x$. Specially, $x=x y x=0$ is provided. This means that $S_{S}=\{0\}$.

(5) If $a \in S_{S}$, then $a S a=0$. Since $a^{3}=0, a$ is a nilpotent element.

(6) If $0 \neq a \in S_{S}$, then $a a a=0$. This equation can be written as $a(a a)=0$ and $(a a) a=0$. Since $0 \neq a$, if $a a=0$, then $a$ is a zero divisor element. On the other hand, if $a a \neq 0$, then $a$ is also a zero divisor element specific with $a^{2}$.

Using the above Lemma 1, it is easy to see that the following corollary.

Corollary 1. For semigroup $S$, the following holds true:

(1) There is no idempotent element other than zero in $S_{S}$

(2) There is no regular element other than zero in $S_{S}$

(3) Every element in $S_{S}$ is nilpotent element

(4) Every element in $S_{S}$ is zero divisor element

Now, we will define different type of $\left|S_{S}\right|$-semigroups.

Definition 2. Let $S$ be semigroup with zero and $S \neq S_{S}$.

(1) $S$ is called the $\left|S_{S}\right|$-idempotent semigroup if every element of $S-S_{S}$ is idempotent

(2) $S$ is called the $\left|S_{S}\right|$-regular semigroup if every element of $S-S_{S}$ is regular

(3) $S$ is called the $\left|S_{S}\right|$-reduced semigroup if $S-S_{S}$ has no nilpotent element

(4) $S$ is called the $\left|S_{S}\right|$-nonzero divisor semigroup if $S-$ $S_{S}$ has neither a left nor a right zero divisor

First, let us mention the basic characteristics properties of these newly defined semigroups. The following results can be easily seen using the definitions.

(1) If $S=\{0\}$, then $S_{S}=\{0\}=S$. In this case, since $S-S_{S}=\varnothing$, definitions would be meaningless for the zero semigroup. Similarly, $S-S_{S}=\varnothing$ is provided in case of $S=S_{S}$. So, in this case too, the definitions would be meaningless.

(2) For the elements of a semigroup, "if $a$ is an idempotent element, then $a$ is regular element," "if $a$ is a nilpotent element, then $a$ is a zero divisor element," 
and "if $a$ is a nilpotent element, then $a$ is not an idempotent element" and conditions are always provided. Using these conditions, the following properties can be easily seen.

$$
\begin{aligned}
& \text { If } S \text { is }\left|S_{S}\right| \text { - idempotent semigroup, then } S \text { is }\left|S_{S}\right| \text { - regular semigroup, } \\
& \text { If } S \text { is }\left|S_{S}\right| \text { - idempotent semigroup, then } S \text { is }\left|S_{S}\right| \text {-reduced semigroup, } \\
& \text { If } S \text { is }\left|S_{S}\right| \text { - nonzero divisor semigroup, then } S \text { is }\left|S_{S}\right| \text { - reduced semigroup. }
\end{aligned}
$$

Additional conditions for providing other directions of relations will be investigated in our study

(3) It is clear that, if $S$ is an idempotent (regular, reduced, and nonzero divisor) semigroup, then $S$ is a $\left|S_{S}\right|$-idempotent (regular, reduced, and nonzero divisor) semigroup

We will give examples of each of these four semigroups before proceeding with conclusions. It can also be seen from these examples that the above relations are one sided.

Example 1. Let the operation table of the semigroup $S$ be given as follows.

$$
\begin{array}{lllll}
\cdot & 0 & a & b & c \\
0 & 0 & 0 & 0 & 0 \\
a & 0 & 0 & 0 & 0 . \\
b & 0 & 0 & 0 & 0 \\
c & 0 & 0 & 0 & c
\end{array}
$$

Using the table, we get

$$
\begin{aligned}
S_{S} & =\{0, a, b\}, \\
S-S_{S} & =\{c\} .
\end{aligned}
$$

Since $c^{2}=c$, it is seen that $c$ is idempotent and regular element of $S-S_{S}$. So, $S$ is a $\left|S_{S}\right|$-idempotent and $\left|S_{S}\right|$-regular semigroup. Also, since $c$ is not nilpotent, $S$ is a $\left|S_{S}\right|$-reduced semigroup. However, $c$ is a zero divisor element for $a c=0$ and $c a=0$. Thus, $S$ is not a $\left|S_{S}\right|$-nonzero divisor semigroup.

Example 2. Let the operation table of the semigroup $S$ be given as follows.

$$
\begin{array}{ccccc}
\cdot & 0 & a & b & c \\
0 & 0 & 0 & 0 & 0 \\
a & 0 & b & b & 0 . \\
b & 0 & b & b & 0 \\
c & 0 & 0 & 0 & 0
\end{array}
$$

For the semigroup $S$, it is easy to see that

$$
\begin{aligned}
S_{S} & =\{0, c\}, \\
S-S_{S} & =\{a, b\} .
\end{aligned}
$$

If we investigate elements of $S-S_{S}$, we see that only $b$ is an idempotent and regular element. So, $S$ is not a
$\left|S_{S}\right|$-idempotent or $\left|S_{S}\right|$-regular semigroup. Also, since $a c=$ 0 and $c a=0, a$ and $c$ are the zero divisor elements. Then, $S$ is not a $\left|S_{S}\right|$-nonzero divisor semigroup. On the other hand, it is clear that $a$ and $b$ are not nilpotent elements. So, $S$ is a $\left|S_{S}\right|$-reduced semigroup.

Example 3. Let the operation table of the semigroup $S$ be given as follows.

$$
\begin{array}{lllll}
\cdot & 0 & a & b & c \\
0 & 0 & 0 & 0 & 0 \\
a & 0 & 0 & a & a . \\
b & 0 & 0 & b & b \\
c & 0 & 0 & c & c
\end{array}
$$

Now, it turns out that

$$
\begin{aligned}
S_{S} & =\{0, a\}, \\
S-S_{S} & =\{b, c\} .
\end{aligned}
$$

It is seen that $b$ and $c$ are the idempotent and regular elements of $S-S_{S}$. Then, $S$ is a $\left|S_{S}\right|$-idempotent and a $\left|S_{S}\right|$-regular semigroup. Also, $b$ and $c$ are nonzero divisors. Therefore, $S$ is a $\left|S_{S}\right|$-nonzero divisor semigroup. On the other hand, since $a$ and $b$ are not nilpotent elements, $S$ is also a $\left|S_{S}\right|$-reduced semigroup.

Example 4. Let the operation table of the semigroup $S$ be given as follows.

$$
\begin{array}{lllll}
\cdot & 0 & a & b & c \\
0 & 0 & 0 & 0 & 0 \\
a & 0 & a & b & c . \\
b & 0 & b & 0 & b \\
c & 0 & c & b & a
\end{array}
$$

Using the table, we get

$$
\begin{aligned}
S_{S} & =\{0, b\}, \\
S-S_{S} & =\{a, c\} .
\end{aligned}
$$

If we investigate the elements of $S-S_{S}$, we see that only $a$ is an idempotent element. Then, $S$ is not a $\left|S_{S}\right|$-idempotent semigroup. However, since $a a a=a$ and $c c c=c, a$ and $c$ are the regular elements. So, $S$ is a $\left|S_{S}\right|$-regular semigroup. Also, $a$ and $c$ are not zero divisor or nilpotent elements. Therefore, $S$ is a $\left|S_{S}\right|$-nonzero divisor and a $\left|S_{S}\right|$-reduced semigroup. 
Example 5. Consider the semigroup $(\mathbb{N}, \cdot)$. Since

$$
S_{\mathbb{N}}=\{0\},
$$

we get

$$
\mathbb{N}-S_{\mathbb{N}}=\{1,2,3, \ldots\}
$$

In the set $\mathbb{N}-S_{\mathbb{N}}$, since only 1 is idempotent and regular, $\mathbb{N}$ is not a $\left|S_{\mathbb{N}}\right|$-idempotent or $\left|S_{\mathbb{N}}\right|$-regular semigroup. On the other hand, since there are no zero divisor elements in $\mathbb{N}-S_{\mathbb{N}}, S$ is a $\left|S_{\mathbb{N}}\right|$-nonzero divisor and $\left|S_{\mathbb{N}}\right|$-reduced semigroup.

Let us now give a proposition and an example about characterization of the subgroups of these new semigroups.

Proposition 1. Let $S$ be a semigroup and $A$ be a subsemigroup of S. Then, the following conditions are satisfied.

(1) If $S$ is a $\left|S_{S}\right|$-idempotent (regular) semigroup, then $A$ is a $\left|S_{A}\right|$-idempotent (regular) semigroup

(2) If $S$ is a $\left|S_{S}\right|$-reduced (nonzero divisor) semigroup, then $A$ is a $\left|S_{A}\right|$-reduced (nonzero divisor) semigroup

Proof

(1) If $a \in A-S_{A}$, then $a \in A$ and $a \notin S_{A}$. Thus, we write $a \in S$ and $a \notin S_{S}$. This means that $a \in S-S_{S}$. So, $A-S_{A} \subseteq S-S_{S}$. Therefore, since every element in $S-$ $S_{S}$ is idempotent (regular), every element in $A-S_{A}$ is an idempotent (regular) element. So, $A$ is a $\left|S_{A}\right|$-idempotent (regular) semigroup.

(2) We showed that $A-S_{A} \subseteq S-S_{S}$ in (1). So, since there is no nilpotent (zero divisor) element in $S-S_{S}$, there is no nilpotent (zero divisor) element in $A-S_{A}$. In this case, $A$ is a $\left|S_{A}\right|$-reduced (nonzero divisor) semigroup.

Example 6. Consider the set

$$
M=\left\{\left[\begin{array}{lll}
x & y & 0 \\
0 & x & 0 \\
0 & 0 & x
\end{array}\right] \mid \quad x, y \in \mathbb{R}\right\} .
$$

$M$ is a semigroup with zero element by multiplication operation in matrices. For this semigroup, it is not hard to see that

$$
\begin{aligned}
S_{M} & =\left\{\left[\begin{array}{lll}
0 & y & 0 \\
0 & 0 & 0 \\
0 & 0 & 0
\end{array}\right] \mid \quad y \in \mathbb{R}\right\}, \\
M-S_{M} & =\left\{\left[\begin{array}{lll}
x & y & 0 \\
0 & x & 0 \\
0 & 0 & x
\end{array}\right] \mid \quad x, y \in \mathbb{R}, x \neq 0\right\} .
\end{aligned}
$$

Since there exist $B=\left[\begin{array}{ccc}1 / x & -y / x^{2} & 0 \\ 0 & 1 / x & 0 \\ 0 & 0 & 1 / x\end{array}\right] \in M-S_{M}$, such that $A B A=A$ for each $A=\left[\begin{array}{lll}x & y & 0 \\ 0 & x & 0 \\ 0 & 0 & x\end{array}\right] \in M-S_{M}, M$ is a

$\left|S_{M}\right|$-regular semigroup. On the other hand, the elements of the set $M-S_{M}$ can only be nilpotent elements for $x=0$. So, $M$ is a $\left|S_{M}\right|$-reduced semigroup. Also, it is easy to see that $M$ is a $\left|S_{M}\right|$-nonzero divisor semigroup because there is no zero divisor element in $M-S_{M}$.

Now, let define the subsemigroup,

$$
A=\left\{\left[\begin{array}{lll}
x & 0 & 0 \\
0 & x & 0 \\
0 & 0 & x
\end{array}\right] \mid \quad x \in \mathbb{R}\right\},
$$

of semigroup $M$. From Proposition $1, A$ is a $\left|S_{A}\right|$-regular, $\left|S_{A}\right|$-reduced, and $\left|S_{A}\right|$-nonzero divisor semigroup. Indeed, it is clear that

$$
\begin{aligned}
S_{A} & =\left\{\left[\begin{array}{lll}
0 & 0 & 0 \\
0 & 0 & 0 \\
0 & 0 & 0
\end{array}\right]\right\}, \\
A-S_{A} & =\left\{\left[\begin{array}{lll}
x & 0 & 0 \\
0 & x & 0 \\
0 & 0 & x
\end{array}\right] \mid x \neq 0\right\} .
\end{aligned}
$$

If we investigate $A-S_{A}$ similar to the above, we see that all elements are regular elements, and there are no nilpotent or zero divisor elements.

Obviously, the set $S-S_{S}$ does not have to be a subsemigroup. The following proposition shows that the $S-S_{S}$ set is the subsemigroup with additional conditions for $\left|S_{S}\right|$-semigroup types. In the next Lemma, the characterization of the $S_{S}$ is given for the $\left|S_{S}\right|$-nonzero divisor and $\left|S_{S}\right|$-reduced semigroups.

Proposition 2. Let $S$ be a semigroup. Then, the following conditions are satisfied.

(1) If $S$ is a $\left|S_{S}\right|$-nonzero divisor semigroup, then $S-S_{S}$ is a subsemigroup

(2) If $S$ is a commutative and $\left|S_{S}\right|$-idempotent (regular) semigroup, then $S-S_{S}$ is a subsemigroup

Proof

(1) Let $S$ be a $\left|S_{S}\right|$-nonzero divisor semigroup. If $x, y \in S-S_{S}$, then $x$ and $y$ are the nonzero divisors. Then, element $x y$ is also a nonzero divisor. This gives us $x y \in S-S_{S}$. So, $S-S_{S}$ is a subsemigroup.

(2) Note that product of idempotent elements is an idempotent element, product of regular elements is a regular element, and product of nilpotent elements is a nilpotent element in a commutative semigroup. In this case, if $a, b \in S-S_{S}$, then $a b \in S-S_{S}$ for the 
$\left|S_{S}\right|$-idempotent (regular) semigroup $S$. So, $S-S_{S}$ is a subsemigroup.

Lemma 2. If $S$ is a $\left|S_{S}\right|$-nonzero divisor or $\left|S_{S}\right|$-reduced semigroup, then $S_{S}=\left\{a \in S \mid a^{3}=0\right\}$. In addition, if $S$ is a monoid, then $S_{S}=\left\{a \in S \mid a^{2}=0\right\}$ is provided for $\left|S_{S}\right|$-nonzero divisor or $\left|S_{S}\right|$-reduced semigroup $S$.

Proof. First, let $S$ be a $\left|S_{S}\right|$-nonzero divisor semigroup and $A=\left\{a \in S \mid a^{3}=0\right\}$. The inclusion $S_{S} \subseteq A$ is always provided. Conversely, let us take an arbitrary element $a$ of $A$. Assume that $a \in S-S_{S}$. Since $S$ is a $\left|S_{S}\right|$-nonzero divisor semigroup, $a$ is a nonzero divisor element. From definition of the set $A$, we write $0=a^{3}=a a^{2}$. Using $a$ as a nonzero divisor, we get $a^{2}=0$. Similarly, from the equation $0=a^{2}=a a$, we get $a=0$. However, this result leads us to the $a \notin S-S_{S}$ contradiction. So the assumption is incorrect, and thus, $a \in S_{S}$. Then, $A \subseteq S_{S}$ is provided.

Now, let $S$ be a $\left|S_{S}\right|$-reduced semigroup. Similar to above, $S_{S} \subseteq A$ is always provided. Conversely, if $a \in A$, then $a^{3}=0$. This gives us $a$ is a nilpotent element. Since $S$ is a $\left|S_{S}\right|$-reduced semigroup, there is no nilpotent element in $S-S_{S}$. So, we get $a \in S_{S}$. Hence $A \subseteq S_{S}$ is provided.

In addition, if $S$ is a monoid, then there exist $1_{S} \in S$, such that $a 1_{S} a=a^{2}=0$ for all $a \in S_{S}$. This means that $S_{S} \subseteq A$. Also, similar to the above proof, we obtain $A \subseteq S_{S}$.

In this part of our study, we will give the results about $\left(\mathbb{Z}_{n}, \cdot\right)$ is an $\left|S_{\mathbb{Z}_{n}}\right|-$ semigroup. As it is known, if monoid $S$ has $a^{-1} \in S$, such that $a a^{-1}=a^{-1} a=1$ for $a \in S, a$ is called an unit element. Since $\mathbb{Z}_{n}$ is a monoid with inverse and unit elements, different results can be reached from other semigroups. In [1], studies on domains and reduced rings were given. In the following part, similar results are obtained by different methods for semigroups.

Lemma 3. Let $\left(\mathbb{Z}_{n}, \cdot\right)$ be semigroup of integers modulo $n$. If $n$ is $p^{2}$ for prime number $p$, then $S_{\mathbb{Z}_{n}}=\{\overline{0}, \bar{p}, \overline{2 p}, \ldots, \overline{(p-1) p}\}$. In addition, $S_{\mathbb{Z}_{n}}=\{\overline{0}\}$ for $n=p$.

Proof. Let $n=p^{2}$ for prime number $p$. From Lemma 2, we write $\bar{a}^{2}=\overline{0}$ for an arbitrary element $\bar{a}$ of $S_{\mathbb{Z}_{n}}$. This means that $p^{2} \mid a^{2}$. Since $p$ is prime, we get $p \mid a^{2}$, and so, we obtain $p \mid a$. Then, $a=p k$ for $k \in \mathbb{Z}$. So, we get $S_{\mathbb{Z}_{n}}=\{\overline{0}, \bar{p}, \overline{2 p}, \ldots, \overline{(p-1) p}\}$.

Let $n=p$ be a prime number and $\bar{a}$ of $S_{\mathbb{Z}_{n}}$. Using the same procedure as in the above paragraph, we have $p \mid a$. Since $p$ is a prime number, the only element that can be divided by $p$ is itself. So, we get $a=p$. This result gives us $\bar{a}=\overline{0}$. So, we get $S_{\mathbb{Z}_{n}}=\{\overline{0}\}$.

Theorem 1. Let $\left(\mathbb{Z}_{n}, \cdot\right)$ be semigroup of integers modulo $n$. The following holds true:

(1) For $n>2, \mathbb{Z}_{n}$ is not a $\left|S_{\mathbb{Z}_{n}}\right|$-idempotent monoid

(2) If $n$ is either a prime $p$ or $p^{2}$, then $\mathbb{Z}_{n}$ is a $\left|S_{\mathbb{Z}_{n}}\right|$-regular monoid
(3) $n$ is either a prime $p$ or $p^{2}$ if and only if $\mathbb{Z}_{n}$ is a $\left|S_{\mathbb{Z}_{n}}\right|$-nonzero divisor monoid

(4) If $n=p$ is a prime number, then $\mathbb{Z}_{n}$ is a $\left|S_{\mathbb{Z}_{n}}\right|$-reduced monoid

Proof

(1) Since $S_{\mathbb{Z}_{2}}=\{\overline{0}\}$, we get $\mathbb{Z}_{2}-S_{\mathbb{Z}_{2}}=\{\overline{1}\}$. It is obvious that $\overline{1}$ is an idempotent element. Therefore, $\mathbb{Z}_{2}$ is a $\left|S_{\mathbb{Z}_{2}}\right|$-idempotent monoid.

Now, we consider monoid $\mathbb{Z}_{n}$ for $n>2$. Let $\bar{a}$ be an unit element of $S_{\mathbb{Z}_{n}}$. Then, $\overline{a x a}=\overline{0}$ for all $\bar{x} \in \mathbb{Z}_{n}$. Specially, $\overline{a a}^{-1} \bar{a}=0$ is provided for $\bar{a}^{-1} \in \mathbb{Z}_{n}$. This result leads us to the contradiction $\bar{a}=\overline{0}$. This means that there is no unit element in $S_{\mathbb{Z}_{n}}$. So, every unit element is element of $\mathbb{Z}_{n}-S_{\mathbb{Z}_{n}}$. In this case, if $\overline{1} \neq \bar{a}$ is an unit element in $\mathbb{Z}_{n}-S_{\mathbb{Z}_{n}}, \bar{a}$ is also an idempotent element. However, an unit element different from the identity cannot be an idempotent element. Therefore, we get $\bar{a}=\overline{1}$. So, the assumption is incorrect, and thus, $\mathbb{Z}_{n}$ is not $\left|S_{\mathbb{Z}_{n}}\right|$-idempotent monoid.

(2) We recall that if $g c d(n, a)=1$, then $\bar{a} \in \mathbb{Z}_{n}$ is an unit element. Let $n=p$ be a prime number. Then, we get $S_{\mathbb{Z}_{n}}=\{\overline{0}\}$ from Lemma 3 . In this case, every element in $\mathbb{Z}_{n}-S_{\mathbb{Z}_{n}}=\mathbb{Z}_{n}-\{\overline{0}\}$ is an unit element. Since an unit element is also a regular element, $\mathbb{Z}_{n}$ is a $\left|S_{\mathbb{Z}_{n}}\right|$-regular monoid.

Let $n=p^{2}$ for prime number $p$. Then, we have $S_{\mathbb{Z}_{n}}=$ $\{\overline{0}, \bar{p}, \overline{2 p}, \ldots, \overline{(p-1) p}\}$ from Lemma 3. This means that, if $\bar{a} \in \mathbb{Z}_{n}-S_{\mathbb{Z}_{n}}$, then $g c d(n, a)=1$. So, $\bar{a}$ is an unit element. Since an unit element is also a regular element, $\mathbb{Z}_{n}$ is a $\left|S_{\mathbb{Z}_{n}}\right|$-regular monoid.

(3) Let $n$ be either a prime $p$ or $p^{2}$. We showed that in (2), if $\bar{a} \in \mathbb{Z}_{n}-S_{\mathbb{Z}_{n}}$, then $\bar{a}$ is an unit element. It is well-known that, an unit element cannot be a zero divisor in $\mathbb{Z}_{n}$. Therefore, there is no zero divisor in $\mathbb{Z}_{n}-S_{\mathbb{Z}_{n}}$. So, $\mathbb{Z}_{n}$ is a $\left|S_{\mathbb{Z}_{n}}\right|$-nonzero divisor monoid. Conversely, let $\mathbb{Z}_{n}$ be a $\left|S_{\mathbb{Z}_{n}}\right|$-nonzero divisor monoid. In this case, every element $\bar{a}$ in $\mathbb{Z}_{n}-S_{\mathbb{Z}_{n}}$ is a nonzero divisor. This means that $\bar{a}$ is an unit element, and $g c d(a, n)=1$. These results provide for $n=p$ any prime number. Assume that $n$ is not prime and $n=p k$ for prime number $p$ and for some $1<k<n$. Since $g c d(p, n) \neq 1, \bar{p}$ is a nonunit element. Hence, $\bar{p}$ must be in $S_{\mathbb{Z}_{\eta}}$, and so, $\bar{p}^{3}=\overline{0}$ from Lemma 2. Therefore, we get $n \mid p^{3}$. Since $n=p k$, we write $p k \mid p^{3}$. This means that $k \mid p^{2}$, and so $k \mid p$. Since $1<k<n$, we obtain $k=p$. So, we get $n=p^{2}$.

(4) Let $n=p$ be a prime number. We showed that in (2), if $\bar{a} \in \mathbb{Z}_{n}-S_{\mathbb{Z}}$, then $\bar{a}$ is an unit element. If $a^{n}=0$, then $0=a^{n} a^{-1}=a^{n-1}$. If we continue in a similar way, then we obtain $a=0$ contradiction. Thus, there is no nilpotent element in $\mathbb{Z}_{n}-S_{\mathbb{Z}_{n}}$. This means that $\mathbb{Z}_{n}$ is a $\left|S_{\mathbb{Z}_{n}}\right|$-reduced monoid. 
Example 7. Consider the monoid $\left(\mathbb{Z}_{4}, \cdot\right)$. For this monoid, we get

$$
\begin{aligned}
S_{\mathbb{Z}_{4}} & =\{\overline{0}, \overline{2}\}, \\
\mathbb{Z}_{4}-S_{\mathbb{Z}_{4}} & =\{\overline{1}, \overline{3}\} .
\end{aligned}
$$

From Theorem $1, \mathbb{Z}_{4}$ is not a $\left|S_{\mathbb{Z}_{4}}\right|$-idempotent monoid. Besides that , $\mathbb{Z}_{4}$ is not $\left|S_{\mathbb{Z}_{4}}\right|$-regular and $\left|S_{\mathbb{Z}_{4}}\right|$-nonzero divisor monoid. Indeed, if the elements of $\mathbb{Z}_{4}-S_{\mathbb{Z}_{4}}$ are investigated, it is seen that $\overline{3}$ is not an idempotent element. However, since $\overline{3} \cdot \overline{3} \cdot \overline{3}=\overline{3}$ and $\overline{1} \cdot \overline{1} \cdot \overline{1}=\overline{1}$, these two elements are regular elements. Then, $\mathbb{Z}_{4}$ is a $\left|S_{\mathbb{Z}_{4}}\right|$-regular monoid. Also, since $\overline{1}$ and $\overline{3}$ are not zero divisors or nilpotent elements, $\mathbb{Z}_{4}$ is a $\left|S_{\mathbb{Z}_{4}}\right|$-nonzero divisor and $\left|S_{\mathbb{Z}_{4}}\right|$-reduced monoid.

Example 8. Consider the monoid $\left(\mathbb{Z}_{8}, \cdot\right)$. Now, it turns out that

$$
\begin{aligned}
S_{\mathbb{Z}_{8}} & =\{\overline{0}, \overline{4}\}, \\
\mathbb{Z}_{8}-S_{\mathbb{Z}_{8}} & =\{\overline{1}, \overline{2}, \overline{3}, \overline{5}, \overline{6}, \overline{7}\} .
\end{aligned}
$$

From Theorem $1, \mathbb{Z}_{8}$ is not a $\left|S_{\mathbb{Z}_{8}}\right|$-idempotent monoid. Indeed, only element $\overline{1}$ is idempotent in $\mathbb{Z}_{8}-S_{\mathbb{Z}_{8}}$. Also, since $\overline{2}$ is not regular element, $\mathbb{Z}_{8}$ is not a $\left|S_{\mathbb{Z}_{8}}\right|$-regular monoid. On the other hand, since $\overline{2}$ is zero divisor and nilpotent, $\mathbb{Z}_{8}$ is a not $\left|S_{\mathbb{Z}_{8}}\right|$-nonzero divisor or $\left|S_{\mathbb{Z}_{8}}\right|$-reduced monoid.

In [10], the relation between the regular semigroups and their ideals was investigated and characterization of the regular semigroups was given. We will now investigate the relation of $\left|S_{S}\right|$-regular semigroups with their ideals.

Lemma 4. Let I and $J$ are the nonempty subsemigroups of semigroup $S$. Then, the following properties are provided.

(1) $S_{I} \cap S_{J} \subseteq S_{I \cap J}$

(2) If I is left (right, both sided) ideal, then $S_{S}(I)$ is right (left, both sided) ideal

(3) If I is ideal, then $S_{I}$ is ideal. Specifically, $S_{S}$ is ideal of $S$.

(4) If I and $J$ are ideals, then $S_{S}(I) \cdot S_{S}(J) \subseteq S_{S}(I J)$

(5) If $I$ and $J$ are ideals, then $S_{I} \cdot S_{J} \subseteq S_{I J}$

Proof. We will prove (1), (2), and (4). (3) and (5) are easily seen; similarly,

(1) If $a \in S_{I} \cap S_{J}$, then $a I a=a J a=0$. Since $I \cap J \subseteq I$, we get $a(I \cap J) a \subseteq a I a=0$. So, $a \in S_{I \cap J}$.

(2) Let $I$ be a left ideal and $a \in S_{S}(I), s \in S$. Then, we write $a \in S$ and $a I a=0$. For arbitrary $x \in I$, since $I$ is a left ideal, we have $s x \in I$. So, the equation

$$
(a s) x(a s)=(a(s x) a) s=0 s=0
$$

is satisfied. This means that $a s \in S_{S}(I)$, and so, $S_{S}(I)$ is right ideal.
(4) Let $a b \in S_{S}(I) \cdot S_{S}(J)$. Then, we get $a I a=0$ and $b J b=0$. Since $I$ is an ideal, we obtain $b I J \subseteq I$. So, we get

$$
a b(I J) a b=a(b I J) a b \subseteq(a I a) b=\{0\} .
$$

This result means that $a b \in S_{S}(I J)$. So, $S_{S}(I) . S_{S}(J) \subseteq S_{S}(I J)$ is provided.

Theorem 2. Let $S$ be a commutative semigroup. $S$ is a $\left|S_{S}\right|$-regular semigroup if and only if $(I \cap J)-S_{I \cap J}=I J-S_{I J}$ for every ideals $I$ and $J$.

Proof. Let $S$ be a $\left|S_{S}\right|$-regular semigroup and $x \in(I \cap J)-S_{I \cap J}$. Thus, $x \in I \cap J \subseteq S$ and $x \notin S_{I \cap J}$. Assume that $x \in S_{S}$. From Lemma 1 and Proposition 2, we get $x=0$. This result contradicts with $x \notin S_{I \cap J}$. Then, $x \in S$ and $x \notin S_{S}$. This gives us $x$ as a regular element. Therefore, there exists $y \in S$, such that $x y x=x$. In this equation, since $I$ and $J$ are ideals, we write $x=x(y x) \in I J$. Also, since $x$ is a regular element and using Proposition $1, x \notin S_{I J}$ is provided. Then, we get $x \in I J-S_{I J}$. From this expression, we obtain

$$
(I \cap J)-S_{I \cap J} \subseteq I J-S_{I J} .
$$

On the other hand, if $x \in I J-S_{I J}$, then $x$ is a regular element because $x \in I J \subseteq S$ and $x \notin S_{I J} \subseteq S_{S}$. Also, since $I J \subseteq I \cap J$, we have $x \in I \cap J$. In this expression, using $x \notin S_{I \cap J}$ for regular element $x$, we obtain $x \in(I \cap J)-S_{I \cap J}$. Therefore,

$$
I J-S_{I J} \subseteq(I \cap J)-S_{I \cap J}
$$

is provided. So, we get $(I \cap J)-S_{I \cap J}=I J-S_{I J}$.

To prove the converse, let $(I \cap J)-S_{I \cap J}=I J-S_{I J}$ for right ideal $I$ and left ideal $J$, and let $a \in S-S_{S}$. Let us consider the ideal $(a)=\{a x \mid x \in S\} \cup\{a\}$ by generated $a$. From the hypothesis, we get

$$
(a)-S_{(a)}=((a) \cap S)-S_{(a) \cap S}=(a) S-S_{(a) S}=a S-S_{a S} .
$$

Using $a \notin S_{S}$ and $S_{(a)} \subseteq S_{S}$, we obtain $a \in(a)-S_{(a)}$. Therefore, we have $a \in a S-S_{a S}$. Also, we know that $a S=S a$ for commutative semigroup $S$. From the hypothesis, we get

$$
a S-S_{a S}=(a S \cap S a)-S_{a S \cap S a}=a S S a-S_{a S S a}=a S^{2} a-S_{a S^{2} a} \text {. }
$$

This equation gives us $a \in\left(a S^{2} a-S_{a S^{2} a}\right)$. This means that $a$ is a regular element such that $a=a b a$ for some $b \in S^{2} \subseteq S$. So, $S$ is a $\left|S_{S}\right|$-regular semigroup.

Theorem 3. Let $S$ be a commutative semigroup. $S$ is a $\left|S_{S}\right|$-regular semigroup if and only if $I^{2}-S_{I^{2}}=I-S_{I}$ is provided for each ideal I of $S$.

Proof. Let $S$ be a $\left|S_{S}\right|$-regular semigroup. For ideal $I$, using Theorem 2, we get 


$$
I^{2}-S_{I^{2}}=I I-S_{I I}=(I \cap I)-S_{I \cap I}=I-S_{I} .
$$

Conversely, let $I^{2}-S_{I^{2}}=I-S_{I}$ for any ideal $I$. Then, since $I \cap J$ is an ideal, we get

$$
(I \cap J)-S_{I \cap J}=(I \cap J)^{2}-S_{(I \cap J)^{2}} \subseteq(I \cap J)(I \cap J)-S_{(I \cap J)^{2} .}
$$

In above equation, using $S_{I \cap J} \cdot S_{I \cap J} \subseteq S_{(I \cap J)^{2}}$ from Lemma 4 (5) and $I J \subseteq I \cap J$, we obtain

$$
(I \cap J)-S_{I \cap J}=(I \cap J)^{2}-S_{(I \cap J)^{2}} \subseteq I J-S_{I \cap J} \cdot S_{I \cap J} .
$$

So, using $S_{I J} \cdot S_{I J} \subseteq S_{I J}$, we get

$$
(I \cap J)-S_{I \cap J} \subseteq I J-S_{I J} .
$$

Also, since $I J \subseteq I \cap J$,

$$
I J-S_{I J} \subseteq(I \cap J)-S_{I \cap J}
$$

is provided. Then, $(I \cap J)-S_{I \cap J}=I J-S_{I J}$. From Theorem 2, $S$ is a $\left|S_{S}\right|$-regular semigroup.

Now, let us give the relation between $\left|S_{S}\right|$-regular and $\left|S_{S}\right|$-reduced semigroups. The following example shows that the commutative property in this theorem is necessary.

Theorem 4. If $S$ is a commutative $\left|S_{S}\right|$-regular semigroup, then $S$ is a $\left|S_{S}\right|$-reduced semigroup.

Proof. Let $S$ be a commutative $\left|S_{S}\right|$-regular semigroup, and let $a \in S-S_{S}$ be a nilpotent element. In this case, there exists $n \in \mathbb{Z}^{+}$, such that $a^{n}=0$ and $a^{n-1} \neq 0$. $a$ is also a regular element. Since $S$ is commutative, there exists $b \in S$, such that $a^{2} b=a$. Thus, we get

$$
0=a^{n}=a^{n-2} a^{2}=a^{n-2} a^{2} b=a^{n-2} a=a^{n-1} .
$$

However, this result contradicts $a^{n-1} \neq 0$. Therefore, there is no nilpotent element in $S-S_{S}$. So, $S$ is a $\left|S_{S}\right|$-reduced semigroup.

Example 9. Let the operation table of the semigroup $S$ be given as follows.

$$
\begin{array}{lllllll}
\cdot & 0 & a & b & c & d & e \\
0 & 0 & 0 & 0 & 0 & 0 & 0 \\
a & 0 & a & 0 & c & 0 & 0 \\
b & 0 & 0 & b & 0 & d & 0 \\
c & 0 & 0 & c & 0 & a & 0 \\
d & 0 & d & 0 & b & 0 & 0 \\
e & 0 & 0 & 0 & 0 & 0 & 0
\end{array}
$$

Using the table, we get

$$
\begin{aligned}
S_{S} & =\{0, e\}, \\
S-S_{S} & =\{a, b, c, d\} .
\end{aligned}
$$

Since $c \in S-S_{S}$ is not an idempotent element, $S$ is not a $\left|S_{S}\right|$-idempotent semigroup. On the other hand, since $a a a=a, c c c=c$, and $c d c=c$, every element in $S-S_{S}$ is regular. So, $S$ is a $\left|S_{S}\right|$-regular semigroup. However, $S$ is not a $\left|S_{S}\right|$-reduced semigroup because $c$ and $d$ are the nilpotent elements. Therefore, $S$ is not a $\left|S_{S}\right|$-nonzero divisor either. As shown in this example, in a noncommutative semigroup, the above result is not valid.

Finally, we will adapt one of the well-known results in semigroup theory to $\left|S_{S}\right|$-regular semigroups. An element $x$ of a semigroup $S$ is called inverse element if there exist at least one $y \in S$, such that $x y x=x$ and $y x y=y$. If there is an inverse element with uniqueness for each element of the $S$ semigroup, the $S$ semigroup is called the inverse semigroup. As can be seen in [8], Proposition 2.6, $S$ is an inverse semigroup if and only if $S$ is a regular and the idempotents of $S$ commute with each other. We reach similar results for $\left|S_{S}\right|-$ regular semigroups, and a relation establishes between $\left|S_{S}\right|$-regular semigroups and inverse semigroups.

Lemma 5. Every elements of $S-S_{S}$ are inverse if and only if $S$ is a $\left|S_{S}\right|$-regular semigroup.

Proof. If every elements of $S-S_{S}$ are inverse, then these elements are also regular elements. It is clear that $S$ is a $\left|S_{S}\right|$-regular semigroup.

Conversely, let $S$ be a $\left|S_{S}\right|$-regular semigroup. Then, each element $a \in S-S_{S}$ is regular, and there exists $b \in S$, such that $a b a=a$.

$$
\begin{aligned}
(b a b) a(b a b) & =(b a b)(a b a) b=(b a b) a b=b(a b a) b \\
& =\text { baban da }(b a b) a=(a b a) b a=a b a=a .
\end{aligned}
$$

So, $b a b$ and $a$ are inverse for each other. So, every elements in $S-S_{S}$ are inverse elements.

Theorem 5. Every elements of $S-S_{S}$ are inverse elements with uniqueness if and only if $S$ is a $\left|S_{S}\right|$-regular semigroup and the idempotents of $S$ commute with each other.

Proof. From Lemma 5, every elements of $S-S_{S}$ are inverse if and only if $S$ is a $\left|S_{S}\right|$-regular semigroup. As can be seen in [8], Proposition 2.6, $S$ is an inverse semigroup if and only if $S$ is a regular and the idempotents of $S$ commute with each other. From this property, all idempotent elements of $S$ commute with each other.

\section{Conclusions}

We have shown that some properties of the source of the semiprimeness defined as $S_{S}=\{a \in S \mid a S a=0\}$ for a semigroup $S$ are given. Moreover, the relations of the source of the semiprimeness with idempotent, regular, nilpotent, and zero divisor elements, which are the basis of the new semigroup structures, are investigated. Additionally, we define the $\left|S_{S}\right|$-idempotent semigroup, $\left|S_{S}\right|$-regular semigroup, $\left|S_{S}\right|-$ reduced semigroup, and $\left|S_{S}\right|$-nonzero divisor 
semigroup structures. Thus, the mentioned semigroups are generalized. Also, we give examples for each semigroup. In particular, the monoid $\left(\mathbb{Z}_{n}, \cdot\right)$ is investigated and generalizations are obtained. Furthermore, we adapt some wellknown results in semigroup theory to new semigroup structures. The source of primeness can be investigated in the sense of this article in future works.

\section{Data Availability}

The data used to support the findings of this study are available from the corresponding author upon request.

\section{Disclosure}

This manuscript is an extended and improved version of the abstract named "A Note on the Source of Semiprimeness of Semigroups" presented in the 9th Mas International European Conference on Mathematics.

\section{Conflicts of Interest}

The authors declare that they have no conflicts of interest.

\section{Acknowledgments}

This work was supported by Çanakkale Onsekiz Mart University, the Scientific Research Coordination Unit (project number: FBA-2019-2792).

\section{References}

[1] N. Aydın, Ç. Demir, and D. Karalarlığlu Camcı, "The source of semiprimeness of rings," Communications of the Korean Mathematical Society, vol. 33, no. 4, pp. 1083-1096, 2018.

[2] D. H. Adams, "Semigroups with no non-zero nilpotent elements," Mathematische Zeitschrift, vol. 123, no. 2, pp. 168-176, 1971.

[3] G. W. S. Van Rooyen, "Remarks on (0-minimal) minimal quasi-ideals in (semigroups with 0) rings," Quaestiones Mathematicae, vol. 18, no. 4, pp. 477-485, 1995.

[4] J. Jezek, T. Kepka, and P. Nemec, "Commutative semigroups that are nil of index 2 and have no irreducible elements," Mathematica Bohemica, vol. 133, no. 1, pp. 1-7, 2008.

[5] A. Reinhart, "Structure of general ideal semigroups of monoids and domains," Journal of Commutative Algebra, vol. 4, no. 3, pp. 413-444, 2012.

[6] J. C. Rosales, "Commutative monoids with zero-divisors," Bollettino Unione Matematica Italiana, vol. 8, no. 5, pp. 773-788, 2002.

[7] A. H. Clifford and G. B. Preston, The Algebraic Theory of Semigroups I, AMS Providence, Washington, DC, USA, 1961.

[8] P. A. Grillet, Semigroups: An Introduction to the Structure Theory, Marcel Dekker, Inc., New York, NY, USA, 1995.

[9] Y. S. Park and J. P. Kim, "Prime and semiprime ideals in semigroups," Kyungpook Mathematical Journal, vol. 32, no. 3, pp. 629-632, 1992.

[10] K. Iseki, “A characterization of regular semigroup," Proceedings of the Japan Academy, vol. 32, no. 9, pp. 676-677, 1956. 